\title{
Are Mendeley Reader Counts Useful Impact Indicators in all Fields? ${ }^{1}$
}

\author{
Mike Thelwall, Statistical Cybermetrics Research Group, University of Wolverhampton
}

Reader counts from the social reference sharing site Mendeley are known to be valuable for early research evaluation. They have strong correlations with citation counts for journal articles but appear about a year before them. There are disciplinary differences in the value of Mendeley reader counts but systematic evidence is needed at the level of narrow fields to reveal its extent. In response, this article compares Mendeley reader counts with Scopus citation counts for journal articles from 2012 in 325 narrow Scopus fields. Despite strong positive correlations in most fields, averaging 0.671 , the correlations in some fields are as weak as 0.255 . Technical reasons explain most weaker correlations, suggesting that the underlying relationship is almost always strong. The exceptions are caused by unusually high educational or professional use or topics of interest within countries that avoid Mendeley. The findings suggest that if care is taken then Mendeley reader counts can be used for early citation impact evidence in almost all fields and for related impact in some of the remainder. As an additional application of the results, cross-checking with Mendeley data can be used to identify indexing anomalies in citation databases.

\section{Introduction}

Citation counts and indicators derived from citation counts are used for informal research evaluations and to support formal research evaluations (Wilsdon, Allen, Belfiore, Campbell, Curry, Hill, et al. 2015). The twin rationales for this are that (a) the cited work has often influenced the citing work (Merton, 1973) and (b) citation counts correlate with expert judgements of the value of articles in many fields and so can be appropriate indicators of both value and scholarly impact (Aksnes \& Taxt, 2004; HEFCE, 2015; van Raan, 2006). One of the many limitations of citation counts is that their useful values lag substantially behind the point at which their influence was felt. When a researcher reads an article, it may be several years before a study that it influences is published. This is due to the time needed to conduct the research, write it up and have it refereed and allocated to a journal issue. This time lag is a disadvantage for most research evaluation purposes because the newest articles cannot be evaluated with the aid of citation counts (e.g., Abramo, Cicero, \& D'Angelo, 2011; Campanario, 2011).

The citation lag problem can be solved by using Mendeley reader counts instead of citations for recent articles. Researchers may add an article to their Mendeley library at the time that they read it or even beforehand (Mohammadi, Thelwall, \& Kousha, 2016), which largely removes the publication lag (Maflahi \& Thelwall, 2016, in press; Thelwall \& Sud, 2016; Thelwall, 2017a). Mendeley reader counts are suitable substitutes for citation counts because they correlate highly with them in many fields (Haustein, Larivière, Thelwall, Amyot, \& Peters, 2014; Mohammadi, \& Thelwall, 2014; Thelwall \& Wilson, 2016; Zahedi, Costas, \& Wouters, 2014; Zahedi, Costas, \& Wouters, 2017), suggesting that they also indicate both scholarly influence and peer quality judgements. The latter has also been demonstrated directly (HEFCE, 2015). Like all altmetrics (Priem, Taraborelli, Groth, \&

\footnotetext{
${ }^{1}$ Thelwall, M. (in press). Are Mendeley reader counts useful impact indicators in all fields? Scientometrics, doi:10.1007/s11192-017-2557-x
} 
Neylon, 2011), they can also be gathered automatically for sets of articles, making them a convenient data source.

The main disadvantage of Mendeley reader counts in this role is that they can be gamed and so should not be used for evaluations when there is the possibility and incentive to manipulate them in advance (e.g., Wouters \& Costas, 2012). A theoretical disadvantage of Mendeley is that most researchers do not use it. From one survey, $8 \%$ of scientists and engineers have joined and $5 \%$ in the social sciences, arts and humanities (Van Noorden, 2014). This is not a problem in practice, however, because reader counts tend to be higher than citation counts for recent articles (Thelwall, 2017a) and, as mentioned above, the two tend to correlate. From these percentages, the number of readers of a social sciences paper could be estimated by multiplying its Mendeley reader count by $1 / 0.05=20$ and for a science paper multiplying by $1 / 0.08=12.5$. These are very approximate figures, however, which obscure field differences and differences between faculty and students. There may be some fields that are exceptions with low Mendeley uptake, such as astrophysics (Bar-Ilan, 2014). The questionnaire from which the figures are derived is also not fully representative of the readers of academic articles.

The relationship between Mendeley reader counts and both peer review quality judgements and citation counts vary by discipline. The Spearman correlation between Mendeley reader counts and peer judgements for journal articles varies between -0.073 (Music, Drama, Dance and Performing Arts, $n=90$ ) and 0.441 ( $n=2070$, Clinical Medicine) for the selected mostly high quality non-review articles submitted to the 36 UK Research Excellence Framework categories (HEFCE, 2015), for example. Similarly, correlations with citation counts vary between 0.36 (Religion: Mohammadi \& Thelwall, 2014) and 0.78 (Anatomy: Thelwall \& Wilson, 2016), at least. In both cases, significant positive correlations indicate that Mendeley reader counts partially reflect both peer review judgements and citation impact. Weaker correlations suggest that other factors also influence the magnitude of Mendeley reader counts (see also: Thelwall, 2016b). For example, they may be biased/skewed towards papers of interest to younger researchers (Mohammadi, Thelwall, Haustein, \& Larivière, 2015), to people in countries that use Mendeley more (Thelwall \& Maflahi, 2015) or in favour of papers that attract non-academic Mendeley users (e.g., students).

The above evidence of disciplinary differences in the relationship between citation counts and Mendeley reader counts does not give a recipe to work out the merits of Mendeley for any individual field because they have all either used broad categories (e.g., five comprehensive categories in: Zahedi, Costas, \& Wouters, 2017) or sampled narrower categories (e.g., 14 broad categories of articles with PubMed IDs and 119 NSF specialities within this set: Haustein, Larivière, Thelwall, Amyot, \& Peters, 2014). To fill this gap, the current article assesses the relationship between Mendeley readers and Scopus citations with a comprehensive set of narrow field categories.

\section{Research questions}

The goal of this paper is to create a comprehensive set of narrow field correlations between citation and reader counts to help research evaluators to assess the strength of Mendeley reader counts as a citation impact indicator in any given area. This is driven by the following research questions.

1. Is the relationship between Mendeley reader counts and traditional scholarly impact, as reflected by citation counts, strong in all fields? 
2. In which fields are Mendeley reader counts most and least common, relative to citation counts?

\section{Methods}

The research design was to gather data from publications from all fields from a single year and compare their citation and reader counts for RQ1 and evaluate Mendeley reader count averages for RQ2.

\section{Data}

Publications were selected from the year 2012 to give a substantial amount of time, five years, to attract citations whilst not being too close to the launch date of Mendeley. The latter ensures that there were substantial numbers of users when the articles were published. Mendeley is most valuable as a source of early impact data for more recent articles but using older data gives more statistically powerful insights into the underlying relationship between citation counts and reader counts. Scopus was chosen as the data source in preference to the Web of Science (WoS) because it has a finer-grained field classification scheme. It also has wider coverage of academic journals (Moed \& Visser, 2008; Mongeon \& Paul-Hus, 2016). Google Scholar has even wider coverage but lacks an effective classification scheme and a method to automatically download all papers from a field (Halevi, Moed, \& Bar-llan, 2017). The new Microsoft Academic has similar coverage to Google Scholar but also lacks an effective field classification scheme (Harzing \& Alakangas, 2017; Hug, Ochsner, \& Brändle, 2017).

Scopus has 36 broad field categories and 335 subfields. The most recent (up to) 5000 articles from each field were downloaded during August 2017. The restriction to 5000 is a system limitation. Although it is possible to get an additional (up to) 5000 results (the oldest) from each field with over 5000 records, this was not done because the total number of articles was already very large. Queries such as the following (for subfield 1206, Conservation) were used in each case, restricting the results to documents of type article (excluding reviews, editorials, notes, etc.).

subjmain(1206) AND DOCTYPE(ar) AND SRCTYPE(j)

No journal articles were recorded by Scopus for seven sub-fields in 2012. Three fields with less than 50 articles were also rejected so the final sample included 325 subfields and $1,257,148$ articles. Four fifths (79.9\%) of the articles had DOIs in Scopus.

The Mendeley reader counts were obtained for each article through Mendeley API queries in Webometric Analyst, submitted in August and September 2017. For all articles, matches were found by querying the API with the first author, title and publication year recorded in Scopus. For articles with DOIs, DOI queries were also used to give more complete results (Zahedi, Haustein, \& Bowman, 2014). The final reader count was the sum of the reader counts of all correctly matching articles (for more details, see: Thelwall \& Wilson, 2016). DOIs are not universal in citation databases (Gorraiz, Melero-Fuentes, Gumpenberger, \& Valderrama-Zurián, 2016) and are usually correct (Franceschini, Maisano, \& Mastrogiacomo, 2015).

The above process does not find a Mendeley record for all Scopus articles. This can occur either because nobody has registered the article in Mendeley or because the search processes have not found it due to metadata errors in Scopus or Mendeley, or incomplete search results in Mendeley. The results probably contain a combination of all these reasons and so it is not clear whether missing records should be interpreted as articles 0 readers or 
missing data. It is also not possible to comprehensively check individual missing records within Mendeley because if the above search process does not find them then they are intrinsically difficult to find in Mendeley.

The number of missing records could, in theory, be estimated for each data set. One approach would be to use the knowledge that sets of citation counts from a single field and year tend to follow a discretised lognormal distribution (Thelwall \& Wilson, 2016). This distribution could be fitted to each Mendeley reader count data set to estimate the "correct" number of zeros, using a zero-inflated version of the discretised lognormal distribution (Thelwall, 2016a). This would be ineffective because some Scopus categories contain excess zeros due to the indexing of academic-related magazines (Thelwall, 2016a). This problem could be circumvented by a two stage strategy: a) estimating the number of uncitable articles (natural zeros) in the Scopus citation count data using zero inflated discretised lognormal regression, and b) estimating the number of missing plus uncitable articles from the Mendeley reader count data also using zero inflated discretised lognormal regression, and c) subtracting the first from the second to estimate the number of missing articles. This approach has the drawback of using two separate regressions, doubling the potential errors. The errors from this are likely to be relatively large and so it is not a practical solution. Instead the simplifying assumption was made that all missing Mendeley data represents uncited articles, but the results cross-checked with the converse assumption that all missing Mendeley data represents articles with an unknown number of Mendeley readers (i.e., genuine missing data).

\section{Analysis}

The relationship between Mendeley reader counts and Scopus citation counts was assessed using Spearman correlation tests. Spearman rather than Pearson correlations were used because the data is skewed. Other factors being equal (e.g., average citation and reader counts, the presence of spurious factors), the strength of the correlation reflects the extent to which the same underlying factors influence both. Although other methods are useful to assess scholarly indicators, correlation tests are the logical first step (Sud \& Thelwall, 2014).

The relative commonness of citation counts compared to Mendeley reader counts was compared based on their geometric means for each field. Geometric means (Thelwall \& Fairclough, 2015; Zitt, 2012) were used rather than arithmetic means because the reader counts (Thelwall \& Wilson, 2016) and citation counts (Merton, 1968) are skewed.

\section{Results}

Scopus citation counts and Mendeley reader counts have positive correlations for journal articles in all academic fields, with the correlations tending to be strong (Table 1). The magnitude of the correlations varies considerably. There are more Mendeley readers than Scopus citations in most fields, with a median of $61 \%$ more. Nevertheless, some fields have more Scopus citations than Mendeley readers.

If documents without records in Mendeley are treated as missing data (Table 2) rather than as having zero readers then the results are similar overall, except that there are relatively more Mendeley readers per paper than citations per paper. The slightly lower correlations overall when missing records are assumed to have no readers (Table 1) suggests that this is the best overall strategy for interpreting Mendeley reader data. The remainder of this section explores the extreme cases in Table 1. 
Table 1. Comparison of Scopus citations and Mendeley reader counts for Scopus-indexed journal articles from 2012 for the 325 Scopus sub-fields with at least 50 articles. For fields with more than 5000 matching articles, the most recent 5000 are included $(1,257,148$ articles altogether). Articles with no Mendeley record found are assumed to have no Mendeley readers. A complete list of values is in the online supplement (doi:10.6084/m9.figshare.5432656).

\begin{tabular}{|l|r|r|l|l|l|l|}
\hline & Articles & $\begin{array}{l}\text { Spearman } \\
\text { correlation }\end{array}$ & $\begin{array}{l}\text { Scopus } \\
\text { citations } \\
\text { (geomean) }\end{array}$ & $\begin{array}{l}\text { Mendeley } \\
\text { readers } \\
\text { (geomean) }\end{array}$ & $\begin{array}{l}\text { Mendeley } \\
\text { - Scopus }\end{array}$ & $\begin{array}{l}\text { Mendey } \\
\text { as of } \\
\text { Scopus }\end{array}$ \\
\hline Min & 87 & 0.255 & 0.25 & 0.27 & -3.27 & $37.6 \%$ \\
\hline Max & 5000 & 0.829 & 22.14 & 31.78 & 20.16 & $452.6 \%$ \\
\hline Median & 4999 & 0.701 & 4.32 & 7.34 & 2.54 & $161.0 \%$ \\
\hline Average & 3868.1 & 0.672 & 4.81 & 8.28 & 3.47 & $181.5 \%$ \\
\hline
\end{tabular}

Table 2. As for Table 1 except that articles with no Mendeley records found were removed $(1,083,964$ articles).

\begin{tabular}{|l|r|r|l|l|r|r|}
\hline & Articles & $\begin{array}{l}\text { Spearman } \\
\text { correlation }\end{array}$ & $\begin{array}{l}\text { Scopus } \\
\text { Citations } \\
\text { (geomean) }\end{array}$ & $\begin{array}{l}\text { Mendeley } \\
\text { readers } \\
\text { (geomean) }\end{array}$ & $\begin{array}{l}\text { Mendeley } \\
\text {-Scopus }\end{array}$ & $\begin{array}{l}\text { Mendeley } \\
\text { as of } \\
\text { Scopus }\end{array}$ \\
\hline Min & 64 & 0.259 & 0.47 & 0.96 & -3.42 & $46.7 \%$ \\
\hline Max & 4970 & 0.818 & 22.27 & 33.67 & 21.74 & $496.3 \%$ \\
\hline Median & 4019 & 0.685 & 5.18 & 10.19 & 4.63 & $189.0 \%$ \\
\hline Average & 3335.3 & 0.658 & 5.59 & 10.94 & 5.34 & $215.1 \%$ \\
\hline
\end{tabular}

\section{Low correlations}

Low correlations can occur for discrete data, such as citation counts, if average values are close to zero because the number of identical values masks the underlying relationship. Thus, as the average number of citations and/or readers per paper decreases, the Spearman correlation can be expected to decrease irrespective of the underlying relationship strength (Thelwall, 2016b). This could explain the three lowest Spearman correlations (Algebra and Number Theory, Geometry and Topology, Classics) since all had Mendeley reader counts below 1.4. The remaining paragraphs discuss cases with correlations under 0.5 but neither low citation counts nor low Mendeley reader counts. For convenience, these were regarded as low if they were below $1 / 3$ of the mean value.

Health Information Management (correlation 0.266, 5.3 citations, 8.4 readers) does not fit this pattern. This category had many records missing from Mendeley. Treating these as missing data rather than unread articles increased the Spearman correlation to 0.453 . The main single cause of missing Mendeley records was the journal Morbidity and Mortality Weekly Report, which had 181 Scopus records with an average of 33 citations each. Only 47 of these were in Mendeley, probably because the articles had no DOIs in Scopus, making their records difficult to query in Mendeley for this journal (missing DOls did not always cause a problem for journals). Theoretical Computer Science (correlation 0.457, 3.6 citations, 3.2 readers) also had several journals entirely or mainly missing from the Mendeley results.

For Signal Processing (correlation 0.301, 3.28 citations, 6.9 readers), removing articles without readers decreases the correlation to 0.259 , suggesting that missing records are not the cause of the low correlation for this sub-field. In this case, the publication with 
Scopus name, "Conference proceedings : ... Annual International Conference of the IEEE Engineering in Medicine and Biology Society. IEEE Engineering in Medicine and Biology Society. Conference" had 738 out of 1595 records with zero citations, only 41 of which had no Mendeley readers. For example, "DataHigh: graphical user interface for visualizing and interacting with high-dimensional neural activity" had 141 readers but no Scopus citations. A search for it in Scopus found three copies. One was from a journal and two were copies of the conference record with different names (the other version was, "Proceedings of the Annual International Conference of the IEEE Engineering in Medicine and Biology Society, EMBS"). Other cases investigated all found at least two records for the two different versions of the conference paper. Thus, in this case the accidental double indexing of the conference seems to have resulted in many citations to its papers being lost. Since it is a conference, its papers are also not journal articles. Computer Vision and Pattern Recognition (correlation 0.442, 4.03 citations, 9.29 readers) included the same conference proceedings as Signal Processing, with the same cause of lower correlations. The same applies to Biomedical Engineering (correlation 0.475, 2.87 citations, 7.58 readers).

Sensory Systems (correlation $0.484,7.69$ citations, 14.31 readers) had a similar problem. Most Journal of Vision articles had been indexed twice in Scopus, with citation counts for each copy of the article apparently being split between both versions. This gave a Spearman correlation of $0.387(n=242)$ for one version and $0.406(n=110)$ for the other, reducing the category average.

Drug Guides (correlation $0.472,1.53$ citations, 6.53 readers) had an unusually high proportion of Mendeley readers classified as Master's degree students (22\%). In this context, educational applications can influence the relationship between citations and readers. For example, it is easy to imagine that, "Two sides to every story: A phenomenological exploration of the meanings of clinical supervision from supervisee and supervisor perspectives" with 22 readers but only 1 citation, is a useful classroom article. The same issue applied to Decision Sciences (miscellaneous) (correlation 0.451, 1.76 citations, 4.32 readers) with 21\% Master's degree students and Equine (correlation 0.493, 5.06 citations, 14.89 readers) with 19\% Master's degree students. The educational skewing was exacerbated by the journal Equine Veterinary Education, which had a high ratio of Mendeley readers per Scopus citation (12.3, double any other Equine journal). Equine also had $13 \%$ Mendeley readers classified as "Other" occupations, which may have been practicing veterinarians or horse riders/owners.

Economics, Econometrics and Finance (all) (correlation 0.482, 1.63 citations, 7.71 readers) had almost $50 \%$ of readers in education (30\% undergraduates, $19 \%$ Master's degree students, $1 \%$ librarians) as well as many magazine-style journals and regional journals without DOls and with over $50 \%$ of articles not found in Mendeley. Thus, educational uses and journal indexing errors combined in this category.

Immunology and Microbiology (miscellaneous) (correlation 0.499, 6.10 citations, 6.52 readers) had a high proportion of first authors from China (48\%) and Japan (18\%). In comparison, only $1 \%$ of readers were from China and $4 \%$ from Japan (19\% were from the USA compared to $9 \%$ of authors). This category had one dominant journal, Experimental and Therapeutic Medicine, with 369 out of the 463 articles. The reason for the weaker correlation in this category therefore seems to be that many topics are primarily of interest in China, and presumably receive citations from Chinese authors but receive few readers because Mendeley is little used in China. For example, "Stellera chamaejasme L. extract induces apoptosis of human lung cancer cells via activation of the death receptor- 
dependent pathway" has 8 Scopus citations, all from Chinese authors, but no Mendeley readers. Stellera chamaejasme is a plant from Tibet. Generalising, correlations in a category can be lowered by the inclusion of topics that are mainly of interest in nations that rarely use Mendeley.

In summary, the main causes of lower correlations are technical: indexing errors or low citation counts and/or reader counts. There is no evidence that Mendeley reader counts do not have a strong underlying relationship with citation counts in any fields, except for sub-fields with high educational uses, as reflected by large proportions of student readers, or sub-fields with a high proportion of researchers from countries that rarely use Mendeley.

\section{Few Mendeley readers}

The five subjects with the lowest proportion of Mendeley readers compared to Scopus citations included four mathematical areas (Analysis, Algebra and Number Theory, Geometry and Topology, Mathematics (all)) as well as Nuclear and High Energy Physics. The mathematics categories did not contain any obvious anomalies and so there seems to be a low uptake of Mendeley in mathematics generally. This may be due to mathematics articles being written in the scientific typesetting language LaTeX. Whilst it is possible to export Mendeley references to LaTeX citations ${ }^{2}$, it is not straightforward and LaTeX users may have other referencing solutions, such as JabRef, which uses the LaTeX BibTeX format. The same seems to apply for High Energy Physics. For example, Journal of High Energy Physics requests document submission in TeX, LaTeX or pdflatex.

\section{Many Mendeley readers}

The five categories with at most Mendeley readers per Scopus citation include four health sciences (Drug Guides, Health Informatics, Orthodontics, Chiropractics) and one general research subfield, Research and Theory. The health categories all suggest a strong relationship with professional training and so the high number of Mendeley readers may be due to the value of journal articles in professional training in these areas. For example, $23 \%$ of Mendeley readers of Chiropractics articles were Master's degree students. The Research and Theory category may be added by scholars to support their research methods training without necessarily citing them.

\section{Discussion}

The results are limited by the selection and categorisation of journals by Scopus (Wang \& Waltman, 2016). Many journals are multidisciplinary or publish related research from other fields and so the journal-based categorisation used is a simplification. Moreover, many studies are inherently multidisciplinary and do not fit well within a single category. The difficulty in identifying articles with no Mendeley readers, as discussed above, is also a limitation. Time is also a factor because the extent to which Mendeley is used may change in the future, along with the ways in which it is used and the types of people that use it.

The most similar prior study (Haustein, Larivière, Thelwall, Amyot, \& Peters, 2014) analysed 1.4 million articles and reviews from 2010 to 2012 indexed in both PubMed and WoS, using citation counts and Mendeley reader counts from 2013. The correlations reported between Mendeley and WoS are not directly comparable since they cover multiple years and more recent data. The lowest values, from Arts ( $n=99$, rho=-0.209) and

\footnotetext{
${ }^{2}$ https://blog.mendeley.com/2011/10/25/howto-use-mendeley-to-create-citations-using-latex-and-bibtex/
} 
Humanities ( $n=691$, rho $=0.227$ ) are lower than the lowest reported in the current paper. This is presumably due to the time to collect Mendeley data since correlations are lower for more recent papers (Thelwall \& Sud, 2016). The same explanation applies to the low correlations found for individual NSF specialities (Haustein, Larivière, Thelwall, Amyot, \& Peters, 2014). The lowest correlation specialisms (History, Psychoanalysis, Communication, Veterinary Medicine, Earth and Planetary Science) were not similar to any low correlation specialism in the current paper. Since the current paper does not rely on PubMed, it is presumably more comprehensive for the three non-health specialisms but the reason for the unusually low correlations for the other two is not known.

Compared to a similar broad prior study (Zahedi, Costas, \& Wouters, 2017), the ratio of Mendeley readers to Scopus citations is about the same overall for publications from 2012: it is higher for newer articles (2013) and lower for older articles (2004-2011). The ratio of Mendeley readers to Scopus citations varied by disciplinary area with relatively few readers in Biomedical and Health Sciences and Natural Sciences and Engineering, and relatively many in social sciences and humanities (Zahedi, Costas, \& Wouters, 2017). The current study gives finer-grained information (see online supplement doi:10.6084/m9.figshare.5432656), showing sub-field-based maximum values for the differences and with mathematics being identified as an area with low readership (due to LaTeX) and professional health-related areas having high readership (due to training).

\section{Conclusions}

The results show that Mendeley reader counts have positive correlations with citation counts in all fields of academia and the correlations are strong in most. Plausible explanations could be found for the fields with the weakest correlations and so the evidence is consistent with the underlying relationship being strong in almost all fields. The technical reasons for the weakness include low average numbers of readers per paper, Scopus indexing errors, and an inability to find articles from some disciplines in Mendeley when they do not have a DOI. The last two reasons can be resolved by manually checking the data for Scopus (or Web of Science) errors and by removing journals without DOls and with a high rate of absence from Mendeley. If there are low correlations due to low Mendeley reader counts or citation counts, then it is still reasonable to use Mendeley reader counts as a proxy for citation counts but they will be less effective at the level of individual articles. This should not be a problem for applications, such as the calculation of field normalised indicators (Thelwall, 2017b; Waltman, van Eck, van Leeuwen, Visser, \& van Raan, 2011), that average values across large groups.

In a few cases, low positive correlations (below 0.5) occurred for non-technical reasons: education use; the inclusion of topics of interest to many researchers from countries that rarely use Mendeley; and (possibly in the Equine case) professional use of Mendeley. There were also fields with relatively high levels of Mendeley use, suggesting a relatively high proportion of non-publishing readers. These bias Mendeley reader counts away from traditional scholarly impact. The first and last issues could be resolved by subtracting reader counts from undergraduates and Master's degree students or the "Other" category. Alternatively, evaluators may accept that it is reasonable to incorporate a degree of educational or professional impact in the indicators. There does not seem to be a

good solution for the national bias issue since an important part of the Mendeley data (e.g., readers of research of interest primarily in China) is effectively missing. Low uptake of 
Mendeley in mathematics-related disciplines due to LaTeX use does not seem likely to cause the same degree of bias, although it lowers the statistical power of Mendeley reader counts.

The current paper has focused on citations to, and readers of, papers that are five years old at the time of data collection but the primary advantage of Mendeley is for early impact evidence for recently published articles. The correlations for these articles would be much lower than those reported here because of the citation counts being mainly zero. Unless early Mendeley readers are unusual in comparison to later Mendeley readers, then the conclusions above about the underlying strong relationship in most fields between readers and citations still hold. Thus, with this assumption and with the above caveats and exceptions, it would be reasonable to use early reader counts as scholarly impact indicators in almost all fields (for a discussion of publication date issues, see also: Haustein, Bowman, \& Costas, 2015; Maflahi \& Thelwall, in press).

As an additional application of the current paper's results, since Scopus contains some indexing errors, studies that rely upon Scopus citation counts and do not need Mendeley reader counts should consider gathering reader counts, correlating them against citation counts, and investigating field/year combinations with low correlations. This might point to indexing errors that can be dealt with to give more accurate citation count data.

\section{References}

Abramo, G., Cicero, T., \& D’Angelo, C. A. (2011). Assessing the varying level of impact measurement accuracy as a function of the citation window length. Journal of Informetrics, 5(4), 659-667.

Aksnes, D. W., \& Taxt, R. E. (2004). Peer reviews and bibliometric indicators: a comparative study at a Norwegian university. Research Evaluation, 13(1), 33-41.

Bar-llan, J. (2014). Astrophysics publications on arXiv, Scopus and Mendeley: a case study. Scientometrics, 100(1), 217-225.

Campanario, J. M. (2011). Empirical study of journal impact factors obtained using the classical two-year citation window versus a five-year citation window. Scientometrics, 87(1), 189-204.

Franceschini, F., Maisano, D., \& Mastrogiacomo, L. (2015). Errors in DOI indexing by bibliometric databases. Scientometrics, 102(3), 2181-2186.

Gorraiz, J., Melero-Fuentes, D., Gumpenberger, C., \& Valderrama-Zurián, J. C. (2016). Availability of digital object identifiers (DOIs) in Web of Science and Scopus. Journal of Informetrics, 10(1), 98-109.

Halevi, G., Moed, H., \& Bar-llan, J. (2017). Suitability of Google Scholar as a source of scientific information and as a source of data for scientific evaluation - Review of the literature. Journal of Informetrics, 11(3), 823-834.

Harzing, A. W., \& Alakangas, S. (2017). Microsoft Academic is one year old: The phoenix is ready to leave the nest. Scientometrics, 112(3), 1887-1894.

Haustein, S., Bowman, T. D., \& Costas, R. (2015). When is an article actually published? An analysis of online availability, publication, and indexation dates. 15th International Conference on Scientometrics and Informetrics (ISSI2015), 1170-1179.

Haustein, S., Larivière, V., Thelwall, M., Amyot, D., \& Peters, I. (2014). Tweets vs. Mendeley readers: How do these two social media metrics differ? IT-Information Technology, 56(5), 207-215.

HEFCE (2015). The Metric Tide: Correlation analysis of REF2014 scores and metrics (Supplementary Report II to the Independent Review of the Role of Metrics in 
Research Assessment and Management). http://www.hefce.ac.uk/pubs/rereports/Year/2015/metrictide/Title,104463,en.html

Hug, S. E., Ochsner, M., \& Brändle, M. P. (2017). Citation analysis with Microsoft Academic. Scientometrics, 111(1), 371-378.

Maflahi, N. \& Thelwall, M. (2016). When are readership counts as useful as citation counts? Scopus versus Mendeley for LIS journals. Journal of the Association for Information Science and Technology, 67(1), 191-199.

Maflahi, N, \& Thelwall, M. (in press). How quickly do publications get read? The evolution of Mendeley reader counts for new articles. Journal of the Association for Information Science and Technology. doi:10.1002/asi.23909

Merton, R. K. (1968). The Matthew effect in science. Science, 159(3810), 56-63.

Merton, R. K. (1973). The sociology of science: Theoretical and empirical investigations. Chicago, IL: University of Chicago press.

Moed, F., \& Visser, M. S. (2008). Appraisal of citation data sources. http://www.hefce.ac.uk/media/hefce/content/pubs/indirreports/2008/missing/Appra isal\%20of\%20Citation\%20Data\%20Sources.pdf

Mongeon, P., \& Paul-Hus, A. (2016). The journal coverage of Web of Science and Scopus: a comparative analysis. Scientometrics, 106(1), 213-228.

Mohammadi, E., Thelwall, M., Haustein, S., \& Larivière, V. (2015). Who reads research articles? An altmetrics analysis of Mendeley user categories. Journal of the Association for Information Science and Technology, 66(9), 1832-1846.

Mohammadi, E., Thelwall, M. \& Kousha, K. (2016). Can Mendeley bookmarks reflect readership? A survey of user motivations. Journal of the Association for Information Science and Technology. 67(5), 1198-1209. doi:10.1002/asi.23477

Mohammadi, E. \& Thelwall, M. (2014). Mendeley readership altmetrics for the social sciences and humanities: Research evaluation and knowledge flows. Journal of the Association for Information Science and Technology, 65(8), 1627-1638.

Priem, J., Taraborelli, D., Groth, P., \& Neylon, C. (2011). Altmetrics: A manifesto. http://altmetrics.org/manifesto.

Sud, P. \& Thelwall, M. (2014). Evaluating altmetrics. Scientometrics, 98(2), 1131-1143. 10.1007/s11192-013-1117-2.

Thelwall, M. \& Fairclough, R. (2015). Geometric journal impact factors correcting for individual highly cited articles. Journal of Informetrics, 9(2),263-272.

Thelwall, M. \& Maflahi, N. (2015). Are scholarly articles disproportionately read in their own country? An analysis of Mendeley readers. Journal of the Association for Information Science and Technology, 66(6), 1124-1135. doi:10.1002/asi.23252

Thelwall, M. \& Sud, P. (2016). Mendeley readership counts: An investigation of temporal and disciplinary differences. Journal of the Association for Information Science and Technology, 57(6), 3036-3050. doi:10.1002/asi.2355

Thelwall, M. \& Wilson, P. (2016). Mendeley readership altmetrics for medical articles: An analysis of 45 fields, Journal of the Association for Information Science and Technology, 67(8), 1962-1972. doi:10.1002/asi.23501

Thelwall, M. (2016a). Are there too many uncited articles? Zero inflated variants of the discretised lognormal and hooked power law distributions. Journal of Informetrics, 10(2), 622-633. doi:10.1016/j.joi.2016.04.014

Thelwall, M. (2016b). Interpreting correlations between citation counts and other indicators. Scientometrics, 108(1), 337-347. doi:10.1007/s11192-016-1973-7 
Thelwall, M. (2017a). Are Mendeley reader counts high enough for research evaluations when articles are published? Aslib Journal of Information Management, 69(2), 174183. doi:10.1108/AJIM-01-2017-0028

Thelwall, M. (2017b). Three practical field normalised alternative indicator formulae for research evaluation. Journal of Informetrics, 11(1), 128-151. 10.1016/j.joi.2016.12.002

Van Noorden, R. (2014). Scientists and the social networks. Nature, 512(7513), 126-130.

van Raan, A. F. (2006). Comparison of the Hirsch-index with standard bibliometric indicators and with peer judgment for 147 chemistry research groups. scientometrics, 67(3), 491-502.

Waltman, L., van Eck, N. J., van Leeuwen, T. N., Visser, M. S., \& van Raan, A. F. (2011). Towards a new crown indicator: An empirical analysis. Scientometrics, 87(3), 467-481.

Wang, Q., \& Waltman, L. (2016). Large-scale analysis of the accuracy of the journal classification systems of Web of Science and Scopus. Journal of Informetrics, 10(2), 347-364.

Wilsdon, J., Allen, L., Belfiore, E., Campbell, P., Curry, S., Hill, S., et al. (2015). The metric tide: Report of the independent review of the role of metrics in research assessment and management.

http://www.hefce.ac.uk/pubs/rereports/Year/2015/metrictide/Title,104463,en.html

Wouters, P., \& Costas, R. (2012). Users, narcissism and control: tracking the impact of scholarly publications in the 21st century. In: E. Archambault, Y. Gingras, V. Larivière (Eds) Proceedings of the 17th International Conference on Science and Technology Indicators. Montreal: Science-Metrix and OST (Vol. 2, pp. 487-497).

Zahedi, Z., Costas, R., \& Wouters, P. (2014). How well developed are altmetrics? A crossdisciplinary analysis of the presence of 'alternative metrics' in scientific publications. Scientometrics, 101(2), 1491-1513.

Zahedi, Z., Costas, R., \& Wouters, P. (2017). Mendeley readership as a filtering tool to identify highly cited publications. Journal of the Association for Information Science and Technology, 68(10), 2511-2521.

Zahedi, Z., Haustein, S. \& Bowman, T. (2014). Exploring data quality and retrieval strategies for Mendeley reader counts. Presentation at SIGMET Metrics 2014 workshop, 5 November 2014. Available: http://www.slideshare.net/StefanieHaustein/sigmetworkshop-asist2014

Zitt, M. (2012). The journal impact factor: Angel, devil, or scapegoat? A comment on JK Vanclay's article 2011. Scientometrics, 92(2), 485-503. 